\title{
Doppler-related Distortions in TOPS SAR Images
}

\author{
Marc Rodriguez-Cassola, Pau Prats-Iraola, Senior Member, IEEE, Francesco De Zan, Rolf Scheiber, Andreas \\ Reigber, Senior Member, IEEE, Dirk Geudtner, Alberto Moreira, Fellow, IEEE
}

\begin{abstract}
A direct consequence of the TOPS acquisition geometry and the steering in azimuth of the antenna is the time-varying Doppler centroid within bursts. If this fact is not properly accommodated during SAR image formation, undesired distortions both in the azimuth and range dimensions of the focused SAR images may appear. Azimuth distortions are caused by the local mismatch of both squint and topography. Range distortions arise from the inaccurate accommodation of the intrapulse motion of the platform, usually known as the stop-and-go approximation. Conventional spaceborne SAR image formation schemes will be, in general, unable to provide accurate TOPS SAR images, . These distortions are discussed and evaluated for exemplary low Earth orbit (LEO) SAR scenarios. Compensations strategies are presented and validated with TerraSAR-X TOPS data. A discussion of the potential impact on the Sentinel-1 Interferometric Wide Swath and Extra Wide Swath modes (i.e., TOPS) is also given.
\end{abstract}

Index Terms-Synthetic Aperture Radar, TOPS, wide-swath SAR modes, burst-mode acquisitions, TerraSAR-X, Sentinel-1, spaceborne SAR missions.

\section{INTRODUCTION}

T ERRAIN Observation with Progressive Scans (TOPS) is a multi-swath scanning burst mode aiming to provide enhanced image performance when compared to conventional ScanSAR [1], [2]. In the TOPS mode, the antenna azimuth beam is steered (aft to fore) at a constant rate, so that all targets on ground are observed using the entire useful part of the azimuth antenna pattern [1], dramatically reducing the scalloping effect and achieving constant azimuth ambiguities and signal-to-noise ratio (SNR) along azimuth. This azimuth steering of the antenna can be regarded as an inverse spotlight, which originates the TOPS name. The TOPS SAR mode was experimentally implemented and for the first time demonstrated in orbit with TerraSAR-X [3]. On the Sentinel1 mission, the Interferometric Wide Swath mode (IW) and the Extra Wide Swath mode (EW) will be operated as TOPS modes [4]. As an attempt to simulate Sentinel-1 data, the TOPS mode at $\mathrm{C}$ band was demonstrated for the European Space Agency (ESA) using Radarsat-2 during 2013 [5]. The TOPS SAR mode has been selected as an operative SAR imaging mode for the ESA-European Commission (EC) cofunded Sentinel 1 mission [4].

As a result of the scanning of the antenna in azimuth, targets at different azimuth positions within the burst are observed

Manuscript received September X, 2013; revised XXXXX. This work was partially supported by the European Space Agency under contract 4000106648/12/NL/MP.

Marc Rodriguez-Cassola, Pau Prats-Iraola, Francesco De Zan, Rolf Scheiber, Andreas Reigber, and Alberto Moreira are with the Microwaves and Radar Institute of the German Aerospace Center (DLR), Oberpfaffenhofen, Germany. E-mail: marc.rodriguez@dlr.de

Dirk Geudtner is with the European Space and Technology Center of the European Space Agency (ESA), Noordwijk, The Netherlands. under different squint angles, i.e., Doppler centroids. The fact of having an azimuth-varying Doppler has a major impact on the efficient implementation of the range-variant SAR image formation kernel, as shown in [6], [7], [8], [9], [10]. Since the early days of SAR, squinted observations have been recognized as challenging due to the increased sensitivity they show to deviations from the actual geometry of the acquisition [11], [12], [13], [14]. Due to the large variation of the Doppler within the burst, TOPS SAR acquisitions are particularly sensitive to geometrical aspects like time-varying squints, topographic changes, and the motion of the satellite during the transmission of the pulse. Usually, these aspects have been mostly related to other research areas such as airborne SAR [15], [16], [17], bistatic SAR [18], [19], very-high resolution spaceborne SAR [20], [21], and continuous-wave SAR [22]. If not accounted for, as it is usually the case in conventional spaceborne SAR image formation schemes, these deviations from the reference geometry introduce undesired distortions both in the azimuth and range dimensions of the focused SAR images. On the one hand, the distortions resulting from the local mismatch of squint and topography may generate phase and azimuth positioning errors in the TOPS SAR images and interferometric pairs. On the other hand, range distortions arise from the intra-pulse motion of the platform, causing a range skew of the focused TOPS burst image.

The paper is divided as follows. Section II presents the TOPS observation geometry and some relevant aspects of the TOPS SAR image formation process. Section III discusses the distortions in the azimuth dimension of TOPS SAR images caused by the dependence of the matched filter on both squint and topographic height. An exemplary performance evaluation for TerraSAR-X images and interferograms is presented, as well as an efficient algorithm to compensate the resulting distortions. Section IV analyzes the impact of the intra-pulse motion of the satellite, and the distortions caused in the range dimension of the TOPS SAR images if this motion is not compensated. Both effects and compensation algorithms are validated using TerraSAR-X TOPS SAR data. Section V evaluates the impact of the analyzed effects on Sentinel-1 IW and EW modes. The paper concludes in Section VI with a discussion.

\section{THE TOPS MODE AS AN EPITOME OF SQUINTED OBSERVATIONS}

The typical TOPS SAR acquisition geometry is illustrated in Fig. 1; for the sake of compactness, only one subswath has been depicted. A single burst within a subswath starts (at time $\left.t_{\mathrm{a}, \mathrm{burst} 1}\right)$ with the antenna steered from the aft to the fore at the constant rate $k_{\text {rot }}^{\mathrm{TOPS}}$. The value of $k_{\text {rot }}^{\mathrm{TOPS}}$ depends on the desired 

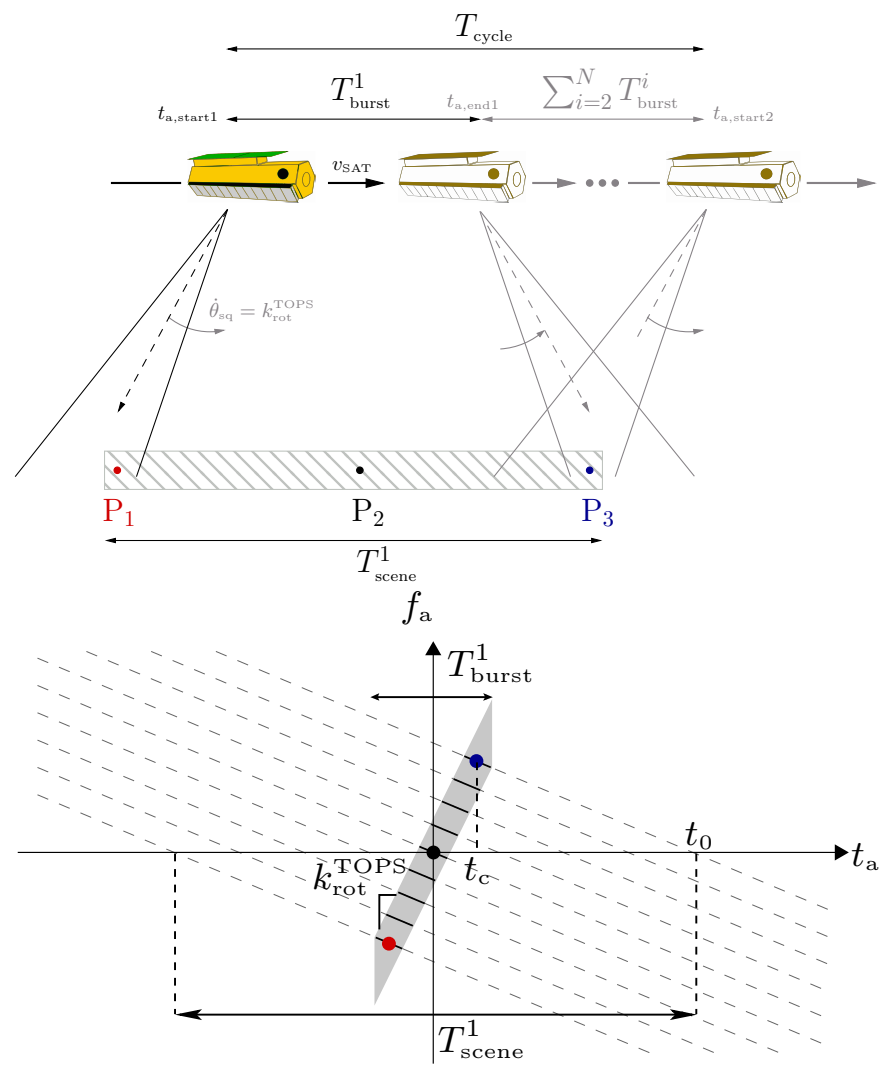

Fig. 1. Schematic diagram of an exemplary TOPS SAR acquisition (top). Time-frequency diagram of a TOPS burst (bottom). For compactness, only one subswath is depicted in the figure.

azimuth resolution, which is somewhat linked to the final swath width of the images [1]. The acquisition usually has a mean zero Doppler centroid and the final squint angle equals in magnitude the initial one. At the end of scanning (in azimuth) the first burst in the first subswath, having a duration of $T_{\mathrm{burst}}^{1}$, all other subswaths are scanned using a similar steering of the azimuth pattern, until the whole swath is covered; the duration of the scanning of all other subswaths is denoted in the figure as $\sum_{i=2}^{N} T_{\mathrm{sub}}^{i}$. After the completion of one burst cycle covering all subswaths, the next burst in the first subswath is transmitted, starting at $t_{\mathrm{a} \text {,start } 2}=t_{\mathrm{a} \text {,start } 1}+T_{\text {cycle }}$. The timefrequency diagram of the acquisition is shown at the bottom of Fig. 1, where the color points on the gray band correspond to the targets $\mathrm{P}_{1}, \mathrm{P}_{2}$, and $\mathrm{P}_{3}$ of the top figure, respectively. As depicted in the bottom figure, $\mathrm{P}_{1}$ is imaged with a negative Doppler centroid, $\mathrm{P}_{2}$ with zero Doppler, and $\mathrm{P}_{3}$ with a positive Doppler centroid. For a constant $k_{\text {rot }}^{\text {TOPS }}$, the Doppler centroid varies linearly with the azimuth position (cf. bottom diagram of Fig. 1). In order to avoid azimuth gaps in the final images, the target $\mathrm{P}_{3}$ is imaged by burst 1 and burst 2 with roughly opposed Doppler centroids (cf. top diagram of Fig. 1), i.e., there exists some overlap between consecutive bursts [1].

Since the beam-center geometry of the targets changes along azimuth, the preferred focusing geometry of TOPS observations will be zero-Doppler. The bottom plot of Fig. 1 shows the position of the targets after SAR image formation in a zero-Doppler geometry, i.e., at the crossing of the dashed gray lines with the $t_{\mathrm{a}}$-axis [9]. Typically, spaceborne SAR image formation kernels (e.g., range-Doppler, omega-K) operate in the Fourier domain and are capable of efficiently accounting for the range-variance of the survey (e.g., range delay, Doppler rate). The efficiency of these kernels is compromised if any azimuth-variance needs be accommodated, i.e., a common matched filter is used for targets placed on the same range coordinate for large blocks in azimuth (e.g., a whole burst). It is, however, well-known that squint, topography and the curvature of the orbits introduce a slight azimuth-variance in the spaceborne SAR survey, which may cause filter mismatch [23], [20]. In Section III, it will be shown that the assumption of azimuth-invariance yields phase and positioning errors in TOPS SAR images as a consequence of the change of the targets range history along azimuth as a function of squint and topographic height.

Although the subsequent analysis has been particularized for TOPS observations, we want to stress that similar effects can be expected for any other SAR squinted (both constant and time-varying) acquisitions. The results presented in this paper could be easily extended to other SAR acquisition modes with constant or varying Doppler centroids, e.g., spotlight, scanSAR, squinted stripmap, and even non-stationary bistatic.

\section{AZIMUTH DISTORTIONS DUE TO SQUINT AND TOPOGRAPHY MISMATCH}

\section{A. Dependence of the effective velocity with squint and topo- graphy}

As sufficiently discussed in the literature, conventional spaceborne SAR processing applies a local approximation of the satellite orbit by a range-dependent straight trajectory [24], [25], [26]. ${ }^{1}$ The range history of a given target can be approximately described by its slant range and the effective velocity of the target, denoted as $v_{\mathrm{e}}$, computed to match the hyperbolical model to the actual geometry of the acquisition; thus, $v_{\mathrm{e}}$ depends on the satellite ephemerides and the target position. Fig. 2 shows the typical scenario for the computation of the effective velocity both considering topography (left) and squint (right). In the left plot, the gray line shows the locus of targets having the same radar coordinates and different heights; in the right plot, the gray line shows the locus of targets having the same slant range coordinate and different positive squints. In typical LEO cases, the variation of the effective velocity of the targets with the topographic height is roughly linear, i.e.,

$$
\frac{\partial v_{\mathrm{e}}}{\partial h} \approx f_{v}\left(\vec{p}_{\mathrm{SAT}}[n], r_{0}\right)
$$

with $f_{v}$ being the constant proportionality factor (i.e., the slope), depending on the orbit ephemerides, $\vec{p}_{\mathrm{SAT}}[n]$, around the azimuth coordinate of the target and on the slant range,

\footnotetext{
${ }^{1}$ This is the usual approach in spaceborne SAR processing, but not essential for the validity of the presented results. However, it allows the derivation of analytical expressions as a function of the effective velocity of the radar. In other cases where the processing kernel must be able to accommodate nonhyperbolical range histories (e.g., bistatic surveys), the basic idea presented here still applies, only the expressions need to be derived as a function of other parameters (e.g., polynomial coefficients) or the distortions be computed numerically. The local straight trajectory model is however accurate for stateof-the-art TOPS SAR systems (e.g., TerraSAR-X, RADARSAT-2, Sentinel-1).
} 


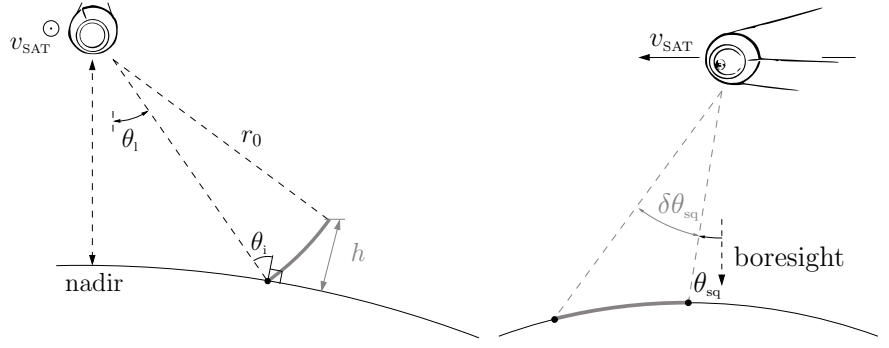

Fig. 2. Cross-track (left) and cross-range imaging geometries (right) of a typical spaceborne SAR survey. The left plot shows the isorange targets located at different topographic heights. The right plot shows isorange targets observed with different squint angles (e.g., the squint excursion is denoted as $\left.\delta \theta_{\mathrm{sq}}\right)$.

TABLE I

KEPLERIAN ELEMENTS OF THE TERRASAR-X ORBIT

\begin{tabular}{cc}
\hline Eccentricity & 0.001 \\
Inclination & $97.44 \mathrm{deg}$ \\
Argument of perigee & $90 \mathrm{deg}$ \\
Ascending node & $88.617 \mathrm{deg}$ \\
Semimajor axis & $6883.513 \mathrm{~km}$ \\
\hline
\end{tabular}

$r_{0}$. Fig. 3 shows the variation of the effective velocity as a function of the topographic height for a TerraSAR-X-like survey over a scene located at 48 deg latitude, 35 deg incident angle. The Keplerian elements of the TerraSAR-X orbit used for the computations are listed in Table I. A total excursion of about $2 \mathrm{~m} / \mathrm{s}$ over a range of $4 \mathrm{~km}$ in topographic height is observed. The value of $f_{v}$ increases with increasing orbit eccentricities, and increasing orbit heights. Fig. 4 shows the value of $f_{v}$ in $\mathrm{mHz}$ for the TerraSAR-X case for different latitudes and incident angles. As expected, the sensitivity to topography relaxes for higher incident angles. Concerning the variation along latitude, a factor six between the smallest and highest values can be observed. Note that the value of $f_{v}$ for the case shown in Fig. 3 is of about $0.5 \mathrm{mHz}$.

For typical LEO cases, the dependence of the effective velocity on the squint angle of the acquisition shows a quadratic

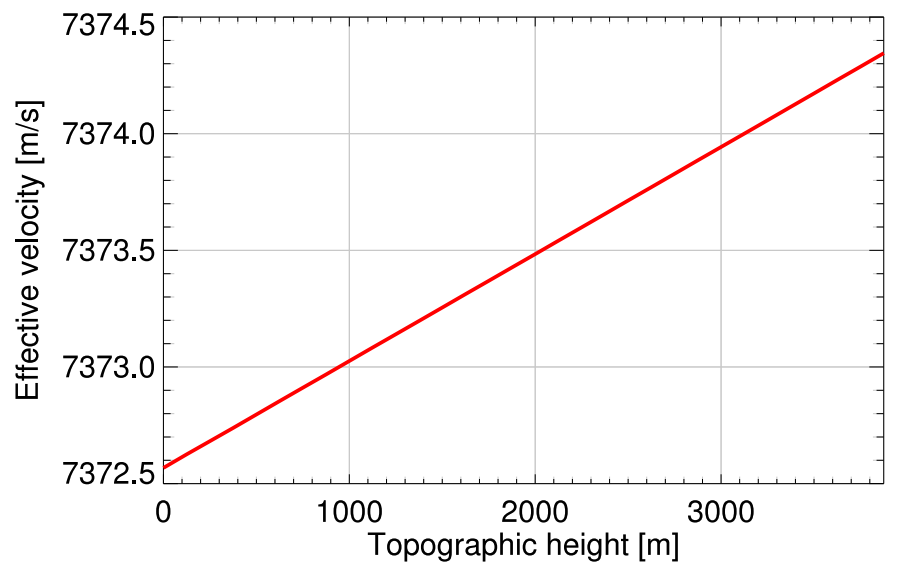

Fig. 3. Change of the effective velocity as a function of topography for TerraSAR-X-like survey over a scene located at $48 \mathrm{deg}$ latitude, $35 \mathrm{deg}$ incident angle.

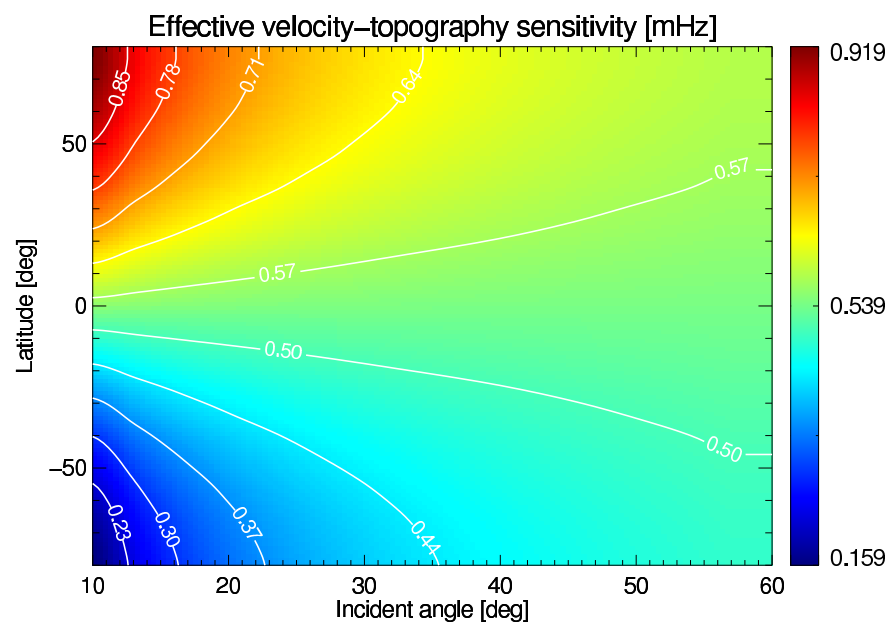

Fig. 4. Values of $f_{v}$ in $\mathrm{mHz}$ for a TerraSAR-X orbit as a function of incident angle and latitude.

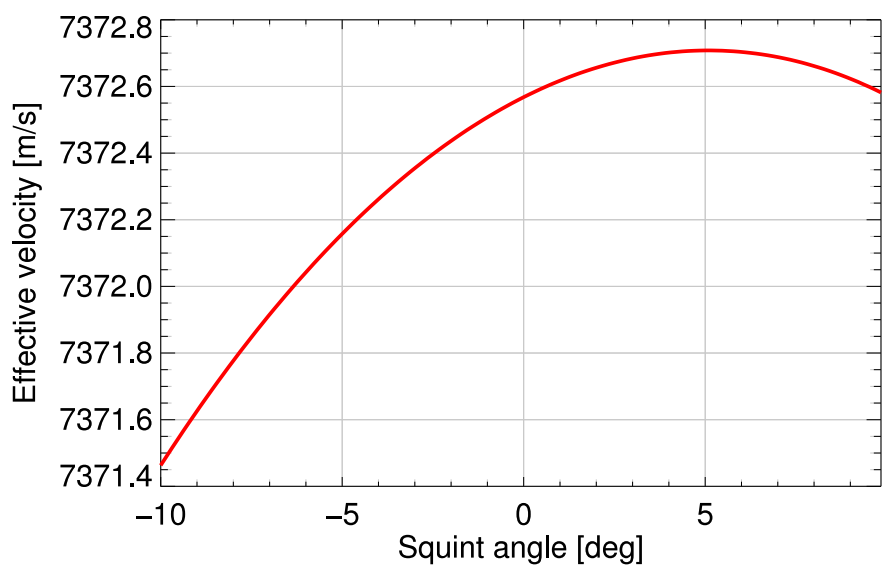

Fig. 5. Change of the effective velocity as a function of squint for a TerraSARX-like survey over a scene located at $48 \mathrm{deg}$ latitude, $35 \mathrm{deg}$ incident angle. Note that the operational steering angles used in TerraSAR-X are kept between $\pm 2.2 \mathrm{deg}$. For illustrative purposes, the observation range of the plot has been extended to $\pm 10 \mathrm{deg}$.

behaviour, i.e.,

$$
\frac{\partial^{2} v_{\mathrm{e}}}{\partial \theta_{\mathrm{sq}}^{2}} \approx k_{\theta}\left(\vec{p}_{\mathrm{SAT}}[n], r_{0}\right)
$$

where $\theta_{\mathrm{sq}}$ is the squint angle and $k_{\theta}$ is the curvature of a parabola. For a TerraSAR-X-like survey over a scene located at $48 \mathrm{deg}$ latitude with $35 \mathrm{deg}$ incident angle, the variation of $v_{\text {e }}$ for a squint angle range between $\pm 10 \mathrm{deg}$ is shown in Fig. 5 . The total variation range is kept below $1.5 \mathrm{~m} / \mathrm{s}$.

B. SAR image formation mismatch due to squint and topography

A typical assumption of Fourier-based SAR focusing kernels is the use of a constant effective velocity for a common range bin (cf. Section II). This translates into the use of the same effective velocity within a large azimuth block of data, typically a whole burst. Since, as discussed in the previous subsection, this effective velocity is depending on topography 
and squint, only a specific isoline ${ }^{2}$ is perfectly matched, i.e., deviations of the real scene topography with respect to the processing isoline result in focusing errors. Assuming the local hyperbolical range history model is valid, a reasonable assumption in the TOPS case, the phase of the matched filter in the wavenumber domain applied to a single range bin within the processing block (e.g., burst) can be expressed as [27]

$$
\Phi_{\mathrm{WN}}\left(f_{\mathrm{r}}, f_{\mathrm{a}} ; v_{\mathrm{e}}\right)=\frac{4 \pi}{\lambda} \cdot r_{0} \cdot \sqrt{\left(\frac{f_{\mathrm{r}}}{f_{0}}\right)^{2}-\left(\frac{\lambda \cdot f_{\mathrm{a}}}{2 \cdot v_{\mathrm{e}}}\right)^{2}},
$$

where $f_{\mathrm{r}}$ is the range frequency, $f_{\mathrm{a}}$ is the azimuth frequency, $\lambda$ is the carrier wavelength, $r_{0}$ is the slant range of the target, and $f_{0}$ is the carrier frequency; in general, $v_{\mathrm{e}}$ is averaged within the processing block using the available geometrical information. The impact of the mismatch of the effective velocity on the range cell migration and secondary range compression terms of (3) is negligible for typical SAR cases. Considering the azimuth compression filter, the phase error along the band can be approximated by

$$
\begin{aligned}
\delta \Phi_{\mathrm{AC}}\left(f_{\mathrm{a}} ; v_{\mathrm{e}}, \delta v_{\mathrm{e}}\right) \approx & -\frac{4 \pi}{\lambda} \cdot r_{0} \cdot \frac{\left(\frac{\lambda \cdot f_{\mathrm{DC}}}{2 \cdot v_{\mathrm{e}}}\right)^{2}}{\sqrt{1-\left(\frac{\lambda \cdot f_{\mathrm{DC}}}{2 \cdot v_{\mathrm{e}}}\right)^{2}}} \cdot \frac{\delta v_{\mathrm{e}}}{v_{\mathrm{e}}} \\
& -2 \pi \cdot \lambda \cdot r_{0} \cdot f_{\mathrm{DC}} \cdot \frac{\delta v_{\mathrm{e}}}{v_{\mathrm{e}}^{3}} \cdot\left(f_{\mathrm{a}}-f_{\mathrm{DC}}\right) \\
& -\pi \cdot \lambda \cdot r_{0} \cdot \frac{\delta v_{\mathrm{e}}}{v_{\mathrm{e}}^{3}} \cdot\left(f_{\mathrm{a}}-f_{\mathrm{DC}}\right)^{2}
\end{aligned}
$$

where the subscript AC stands for azimuth compression, $f_{\mathrm{DC}}$ is the Doppler centroid under which the target is seen, $\delta v_{\mathrm{e}}$ is the deviation of the effective velocity of the actual target with respect to to the one used during azimuth compression. The first term of (4) causes a phase error, the second term a positioning error, and the third term both defocusing and an additional phase error. For typical TOPS cases, the defocusing due to the mismatch of the effective velocity is negligible, ${ }^{3}$ and therefore only the phase error and the positioning error in azimuth will be relevant; the phase error can be approximated by

$$
\delta \phi \approx-\frac{4 \pi}{\lambda} \cdot r_{0} \cdot \frac{\delta v_{\mathrm{e}}}{v_{\mathrm{e}}} \cdot\left[\frac{\left(\frac{\lambda \cdot f_{\mathrm{DC}}}{2 \cdot v_{\mathrm{e}}}\right)^{2}}{\sqrt{1-\left(\frac{\lambda \cdot f_{\mathrm{DC}}}{2 \cdot v_{\mathrm{e}}}\right)^{2}}}-\frac{\lambda^{2} \cdot B_{\mathrm{a}}^{2}}{4 \cdot k \cdot v_{\mathrm{e}}^{2}}\right],
$$

where $k$ is a factor relating the quadratic phase error at the edge of the azimuth processed band to the phase error of the target, which is approximately equal to 3 for a rectangular weighting [26]. Analogously, the azimuth shift of the impulse response takes the value [25], [28]

$$
\delta t_{\mathrm{a}} \approx f_{\mathrm{DC}} \cdot \frac{\delta K_{\mathrm{a}}}{K_{\mathrm{a}}^{2}} \approx-\lambda \cdot r_{0} \cdot f_{\mathrm{DC}} \cdot \frac{\delta v_{\mathrm{e}}}{v_{\mathrm{e}}^{3}},
$$

\footnotetext{
${ }^{2}$ Due to the orbit's and Earth's curvature and the steering in azimuth of the antenna, this line of constant effective velocity shows slight deviations with respect to the constant height contour line.

${ }^{3}$ In the TerraSAR-X case, the quadratic phase error at the edge of the band is smaller than $0.5 \mathrm{deg}$.
}

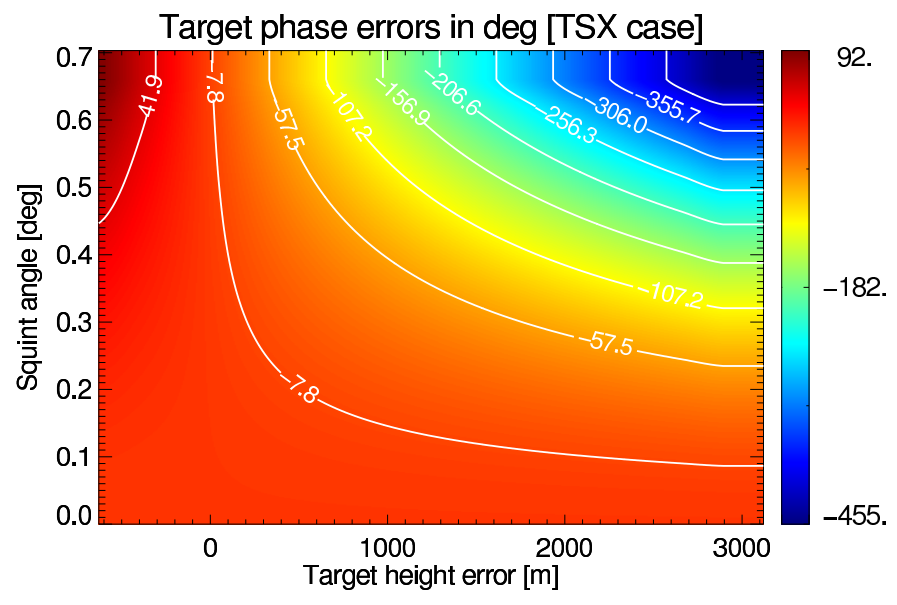

Fig. 6. Target phase error as a function of squint angle and topographic height difference with respect to the reference (processing) level. A Keplerian TerraSAR-X (TSX) like orbit has been used in the computations.

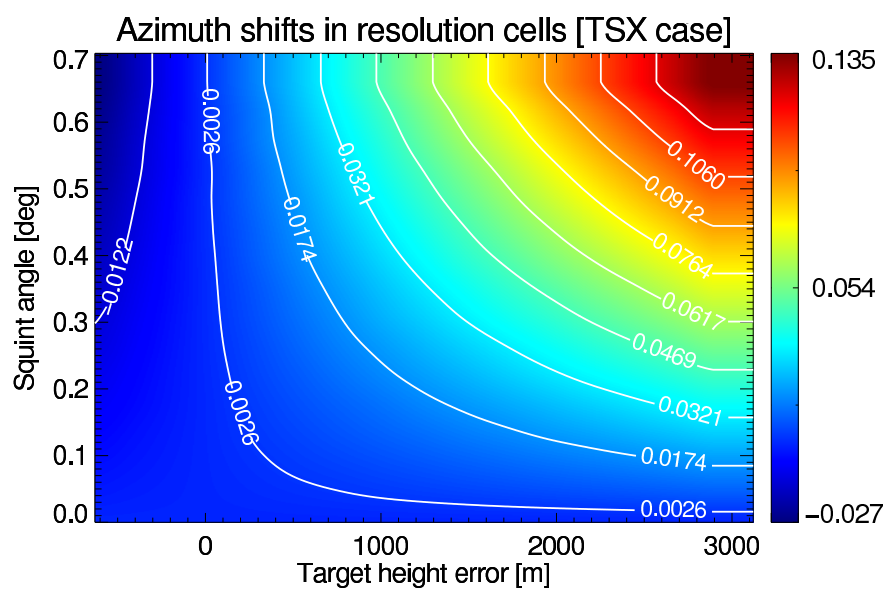

Fig. 7. Azimuth shifts of the focused target as a function of squint angle and topographic height difference with respect to the reference (processing) level. Processed bandwidth of $450 \mathrm{~Hz}$. A TerraSAR-X (TSX) case has been assumed.

where $K_{\mathrm{a}}$ is the Doppler rate of the target. Note that the dependence of the induced shift on $\lambda$ cancels out after the multiplication with the Doppler centroid. Fig. 6 shows the phase error of the focused targets for a typical TerraSAR-X acquisition, with up to $0.7 \mathrm{deg}$ squint angle. The error is displayed as a function of the squint and the topographic height, assuming the reference height for the computation of the processing $v_{\mathrm{e}}$ is $625 \mathrm{~m}$. Significant errors in the phase of the TOPS images are expected for scenes with large topographic variations if the effect is not properly accounted for. Fig. 7 shows the corresponding azimuth shifts (in resolution cells) for the same TerraSAR-X case assuming a processed azimuth bandwidth of $450 \mathrm{~Hz}$. As in the previous case, significant azimuth shifts of up to 0.14 resolution cells (about $2.3 \mathrm{~m}$ ) are expected in TerraSAR-X TOPS SAR images if topography is not locally accommodated. If not compensated, these errors may reduce the geolocation accuracy of TOPS (in general, squinted) SAR images.

In order to validate the model of the aforementioned distortions due to squint and topography mismatch, the overlap 

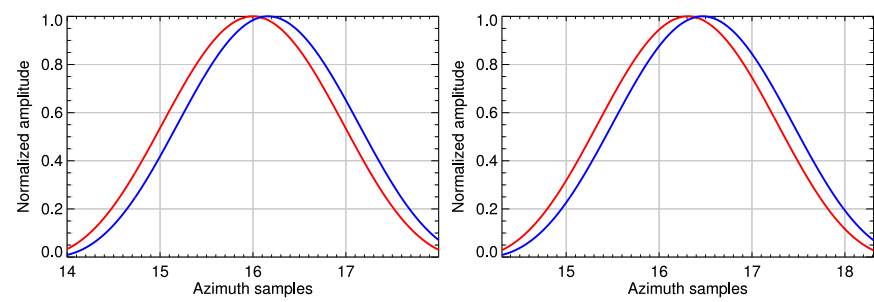

Fig. 8. Interpolated azimuth responses of two corner reflectors located in subswaths 1 (left) and 2 (right). The corner reflectors are located in the overlap region between two consecutive bursts. An ellipsoidal height off by $2 \mathrm{~km}$ has been used during processing. In red, the responses of the first burst; in blue, the responses of the second burst. An azimuth shift of 0.16 pixels is observed.

area between two consecutive bursts of a TerraSAR-X TOPS acquisition has been analyzed. In the burst overlap areas of mean zero-squinted acquisitions, the Doppler centroid of the targets has about the same magnitude and opposite sign; the inter-burst phase error cancels out, whereas the inter-burst azimuth coregistration error takes the form

$$
\delta t_{\mathrm{a}, \mathrm{burst}}=-\lambda \cdot r_{0} \cdot \Delta f_{\mathrm{ovl}} \cdot \frac{\delta v_{\mathrm{e}}}{v_{\mathrm{e}}^{3}},
$$

where $\Delta f_{\text {ovl }}$ is the spectral separation between the Doppler centroids with which the target is seen in the two consecutive bursts, about $6 \mathrm{kHz}$ for TerraSAR-X. An acquisition over DLR in Oberpfaffenhofen with corner reflectors deployed in the burst overlap area was carried out for calibration purposes. The images were first processed using an erroneous effective velocity resulting from averaging over an ellipsoidal height with an offset of $2 \mathrm{~km}$ and no local accommodation of the Doppler centroid; Fig. 8 shows the result of the main lobe in azimuth of the responses of the two corner reflectors for the preceding (red) and following bursts (blue); the azimuth shift of about 0.16 pixels and conform with (7) is clearly visible.

\section{Interferometric error budget}

In this subsection, we discuss the impact of the phase and azimuth positioning errors resulting from the mismatch of the effective velocity on the interferometric performance of TOPS image pairs. Two different cases can be identified: a) two similar TOPS acquisitions with a baseline (e.g., repeator single-pass TOPS acquisitions with full- or half-baselines) where a common squint scan can be assumed and b) the combination of a TOPS SAR image with another source (stripmap, spotlight, scanSAR, or a different TOPS) acquired with a different squint scan. ${ }^{4}$ In the latter cases, errors are expected to be considerably different for master and slave and the interferometric phase error reduces to the expression in (5) and the typical values for TerraSAR-X given in Fig. 6 .

In the case where two similar acquisitions are combined, things improve due to the similarity of the observed phase and positioning errors for both master and slaves images. The Doppler centroids with which the targets are observed

\footnotetext{
${ }^{4}$ Note that in this case, the combination of two different multi-swath acquisitions is of reduced importance since a very small common bandwidth is expected. However, in applications involving the use of multi-mode coherent stacks as PS/CS interferometry the single image distortions become relevant.
}

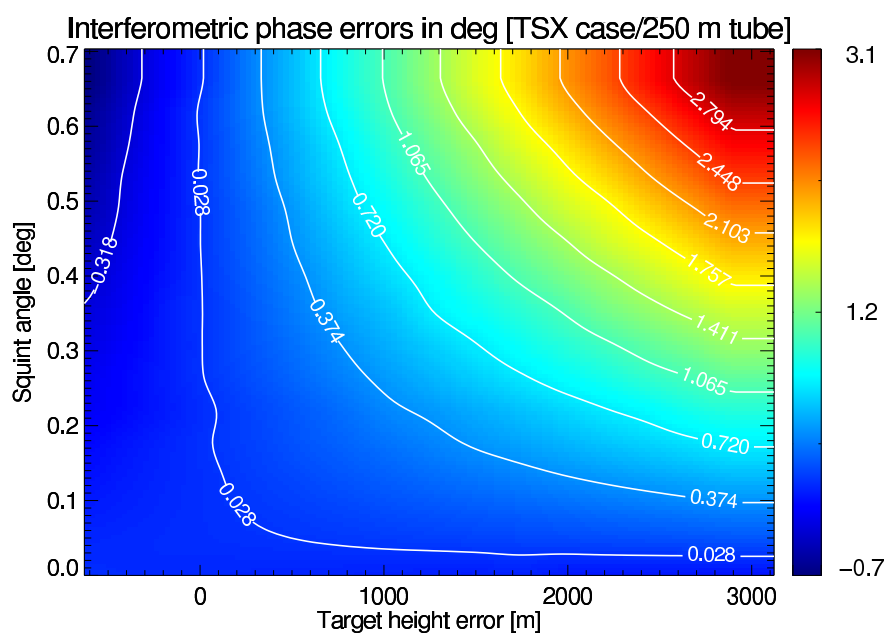

Fig. 9. Typical interferometric phase errors caused by local mismatch of squint and topography for the TerraSAR-X (TSX) case. Orbital tube diameter of 250 $\mathrm{m}$.

are approximately equal. ${ }^{5}$ The resulting interferometric phase error can be approximated as

$$
\delta \phi_{\mathrm{int}} \approx \delta \phi\left(r_{0, \mathrm{~m}}, v_{\mathrm{e}, \mathrm{m}}, \delta v_{\mathrm{e}, \mathrm{m}}\right)-\delta \phi\left(r_{0, \mathrm{~s}}, v_{\mathrm{e}, \mathrm{s}}, \delta v_{\mathrm{e}, \mathrm{s}}\right),
$$

where the subscripts $\mathrm{m}$ and $\mathrm{s}$ stand for master and slave images, respectively. For typical moderate baselines, the values of $v_{\mathrm{e}}$ and $\delta v_{\mathrm{e}}$ for master and slave are similar and (8) can be further approximated as

$$
\delta \phi_{\mathrm{int}} \approx-\frac{4 \pi}{\lambda} \cdot \frac{\left(\frac{\lambda \cdot f_{\mathrm{DC}}}{2 \cdot v_{\mathrm{e}}}\right)^{2}}{\sqrt{1-\left(\frac{\lambda \cdot f_{\mathrm{DC}}}{2 \cdot v_{\mathrm{e}}}\right)^{2}}} \cdot \frac{\delta v_{\mathrm{e}}}{v_{\mathrm{e}}} \cdot\left(r_{0, \mathrm{~m}}-r_{0, \mathrm{~s}}\right)
$$

The coherence loss caused by misregistration can be approximated under the previous assumptions as [29]

$$
\begin{aligned}
\gamma_{\mathrm{a}} & \approx \operatorname{sinc}\left[B_{\mathrm{a}} \cdot\left(\delta t_{\mathrm{a}, \mathrm{m}}-\delta t_{\mathrm{a}, \mathrm{s}}\right)\right] \\
& \approx \operatorname{sinc}\left[\lambda \cdot B_{\mathrm{a}} \cdot f_{\mathrm{DC}} \cdot \frac{\delta v_{\mathrm{e}}}{v_{\mathrm{e}}^{3}} \cdot\left(r_{0, \mathrm{~m}}-r_{0, \mathrm{~s}}\right)\right] ;
\end{aligned}
$$

in typical TOPS cases, though, this coherence loss is negligible, since the shifts are very similar due to the moderate baselines. It might be relevant in other acquisition modes with time-varying Doppler centroids such as sliding spotlight. In the TerraSAR-X TOPS case, and considering an orbital tube with a diameter of about $250 \mathrm{~m}$, the differences of the positioning errors are insignificant and the interferometric phase errors remain small. Fig. 9 shows a typical case of interferometric phase errors assuming the same squint and topographic height range used in Figs. 5 and 6; it can be seen that the interferometric phase errors are kept below 4 deg, even for large topographic variations. In the case of larger baslines, interferometric phase errors due to squint and topography mismatch may become relevant. As an example, Fig. 10 shows an analogous plot for an orbital tube of $3 \mathrm{~km}$; as predicted by (9), the variation range of the interferometric

\footnotetext{
${ }^{5}$ Note that there might be slight changes in the effective Doppler centroids due to the slight lack of parallelism between the orbits of master and slave. This effect can be neglected in typical cases.
} 


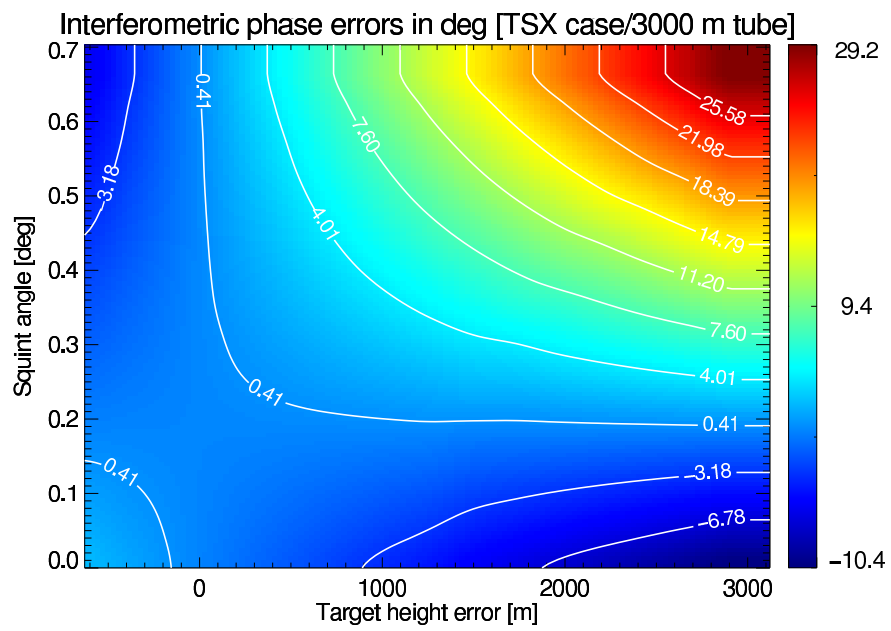

Fig. 10. Typical interferometric phase errors caused by local mismatch of squint and topography mismatch for the TerraSAR-X (TSX) case. Orbital tube diameter of $3000 \mathrm{~m}$.

phase error is increased beyond $40 \mathrm{deg}$. Note that in such a case, reasonable for future interferometric SAR missions, the interferometric phase errors become relevant even in scenes with normal topographic variations.

\section{Post-processing algorithm for squint and topography ac- commodation (PASTA)}

The post-processing algorithm for squint and topography accommodation (acronym PASTA) is a precise yet efficient manner to compensate for an azimuth-variant mismatch within range bins. Inspired in existing motion compensation approaches for airborne SAR data [16], PASTA is capable of achieving excellent results even if the data have been focused neglecting the dependence of the effective velocity with range (e.g., a classical assumption of the canonical omega-K focusing kernel). PASTA is applied after azimuth compression and can be straightforwardly parallelized. The flow of the algorithm is shown in Fig. 11. In the diagram, the effective velocity used in the processing, $\tilde{v}_{\mathrm{e}}$, is range-dependent. The real effective velocities of the scene, represented in the matrix $v_{\mathrm{e}}$, are computed using the actual geometry of the acquisition, i.e., satellite orbit and DEM of the scene. PASTA works as a post-processing approach, i.e., on the focused single look complex data (SLC). The first step is a deramping to set the data on a common frequency reference. The algorithm works with short blocks in azimuth centered on the pixel to be corrected. An azimuth DFT is computed on the block, after which the compensation phase $\delta \Phi_{\mathrm{AC}}$ is applied. Note $\delta \Phi_{\mathrm{AC}}$ is accurately computed by using the actual geometry of the acquisition and not necessarily using the approximation in (4), i.e.,

$$
\begin{aligned}
\delta \Phi_{\mathrm{AC}}= & \frac{4 \pi}{\lambda} \cdot r_{0} \cdot \sqrt{1-\left(\frac{\lambda \cdot f_{\mathrm{a}}}{2 \cdot v_{\mathrm{e}}}\right)^{2}} \\
& -\frac{4 \pi}{\lambda} \cdot r_{0} \cdot \sqrt{1-\left(\frac{\lambda \cdot f_{\mathrm{a}}}{2 \cdot \tilde{v}_{\mathrm{e}}}\right)^{2}} .
\end{aligned}
$$

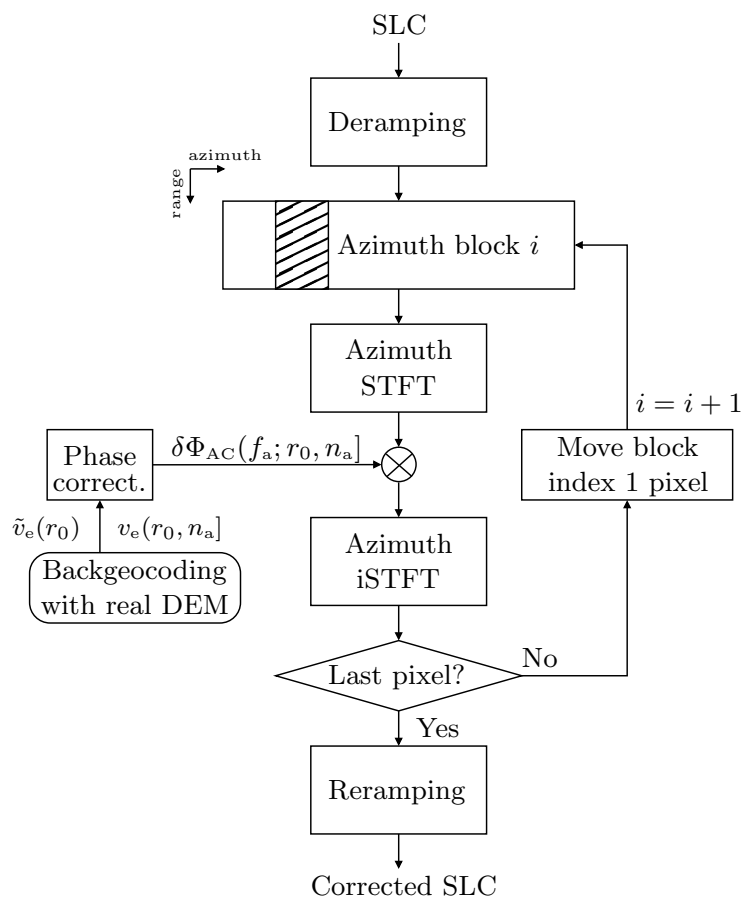

Fig. 11. Block diagram of the post-processing algorithm for squint and topography accommodation (PASTA). The acronym STFT stands for shorttime Fourier transform.
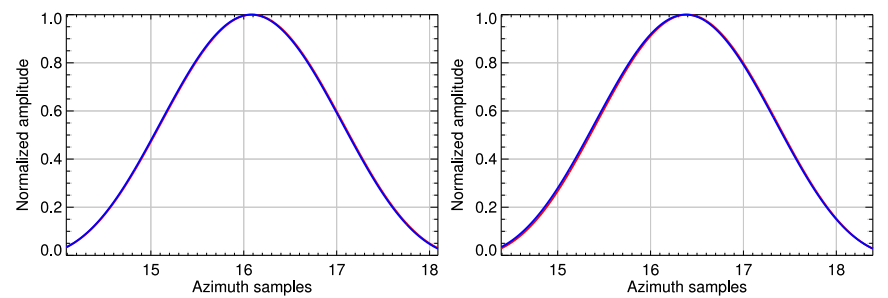

Fig. 12. Interpolated azimuth responses of two corner reflectors located in subswaths 1 (left) and 2 (right). The corner reflectors are located in the overlap region between two consecutive bursts. An ellipsoidal height off by $2 \mathrm{~km}$ was used during processing and PASTA was applied as a post-processing step. In red, the responses of the first burst; in blue, the responses of the second burst. The azimuth shift of 0.16 pixels shown in Fig. 8 has been successfully corrected.

This residual correction is performed in the range-Doppler domain. After this, the whole azimuth bin is corrected. Once all pixels are corrected, the data are reramped to their original frequency reference. The algorithm is easily parallelizable and runs in comparable times as the focusing of the burst itself using [9]. For the validation of the algorithm, the same TerraSAR-X TOPS data over Oberpfaffenhofen have been used; the images have been processed using a wrong ellipsoidal height off by $2 \mathrm{~km}$ with respect to the true one. Once PASTA is applied on the wrongly processed data, the shifted responses of the corner reflectors appear at the same azimuth position in the two consecutive bursts as it is shown in Fig. 12.

\section{E. Experimental results with TerraSAR-X data}

Experimental TOPS data acquired by TerraSAR-X over the Atacama desert, in Chile, have been used to put forward the 

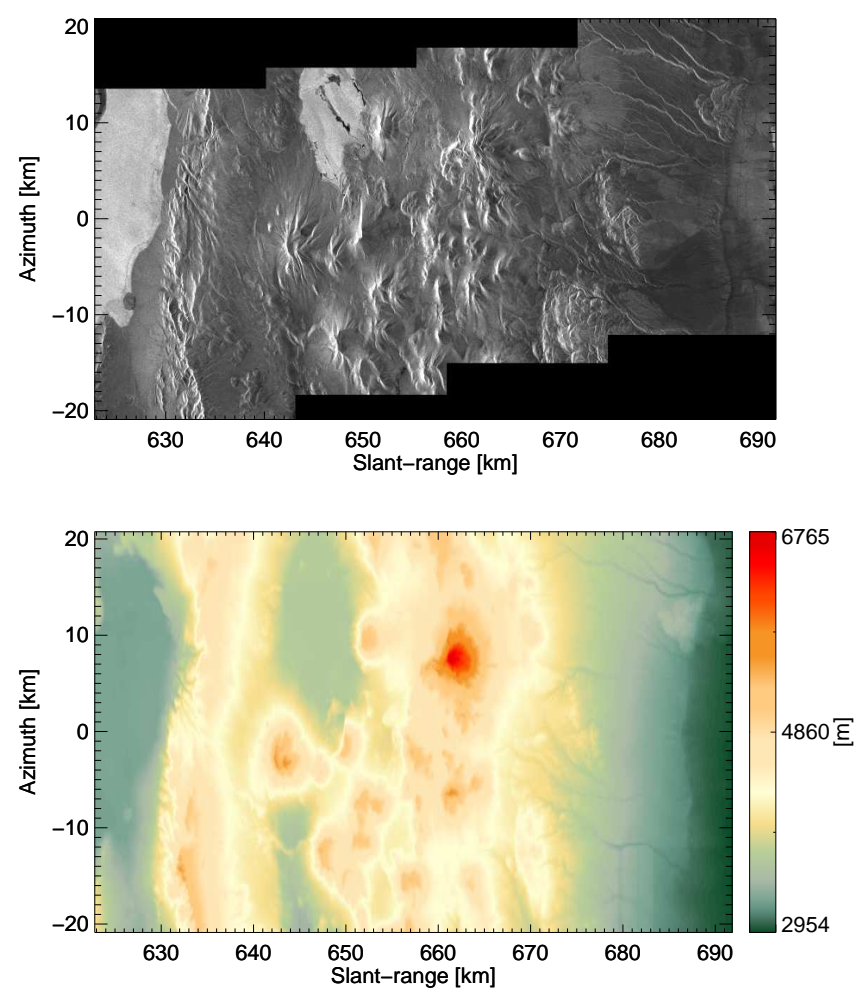

Fig. 13. TerraSAR-X TOPS reflectivity image (3 bursts and 4 subswaths) acquired over the Atacama desert, Chile and corresponding DEM (bottom).

relevance of the presented analysis. Fig. 13 shows the reflectivity image (top) of the first 4 bursts and the corresponding DEM (bottom); note the topographic variations within the scene are over $3500 \mathrm{~m}$. To better illustrate the results, a processing over an average height (ellipsoidal height) of 3700 $\mathrm{m}$ is used as a reference; PASTA is then applied on the data. ${ }^{6}$ Fig. 14 shows the comparison between the reference SLC image processed on the average ellipsoid and the corrected SLC after applying PASTA. The top image shows the phase errors and the bottom image shows the estimated azimuth shifts. Both agree with the values obtained in Figs. 6 and 7. Fig. 15 shows the interferometric errors by using a repeatpass interferometric pair. The figure shows the phase difference between the interferogram computed with the reference SLCs processed on the average ellipsoid, and the interferogram computed with the reference SLCs corrected with PASTA. Values are comparable with those of Fig. 9.

\section{RANGE DISTORTIONS CAUSED BY THE INTRA-PULSE MOTION OF THE PLATFORM}

\section{A. The stop-and-go approximation}

In conventional spaceborne SAR processing, the continuous motion of the platform is usually neglected, an approximation

\footnotetext{
${ }^{6} \mathrm{~A}$ more realistic processing takes into account the real DEM of the scene capable of a range-dependent, azimuth-averaged topographic accommodation which might result in smaller errors than the ones presented here. This however does not affect the illustratory purpose of this subsection.
}
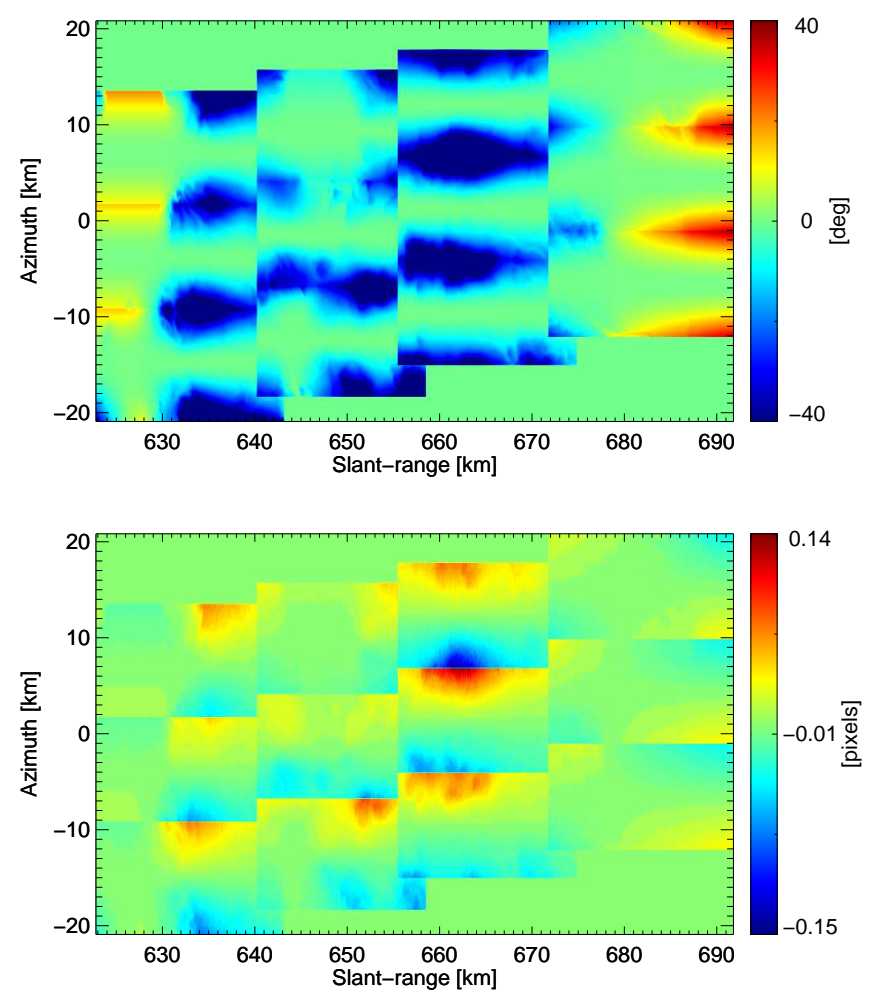

Fig. 14. Phase difference (top) between the reference SLC processed on the ellipsoid before and after PASTA. Corresponding azimuth shifts (bottom).

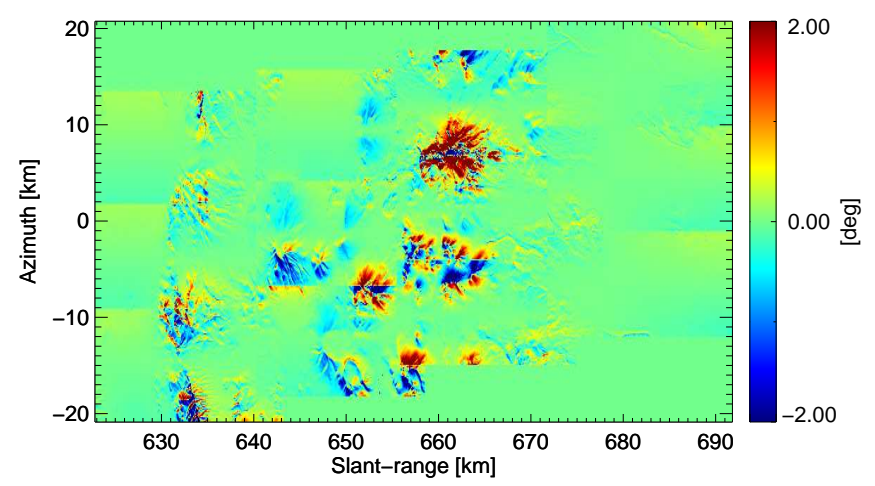

Fig. 15. Interferometric phase errors between the reference pair processed on the ellipsoid before and after PASTA.

known as stop-and-go [26]. This approximation has two effects: a) one in slow-time due to the motion of the satellite between transmission and reception of the transmitted pulses [30]; and b) another in fast-time due to the motion of the satellite during the transmission and reception of the chirp signal itself. ${ }^{7}$ For typical cases, the slow-time effect is roughly constant within the range bin and can be easily compensated with a range-dependent azimuth shift after range cell migration correction [30]. In a linear approximation, the motion of the

\footnotetext{
${ }^{7}$ As an example, during the transmission of a pulse of $50 \mu \mathrm{s}$, TerraSAR-X moves about $38 \mathrm{~cm}$ in the azimuth direction.
} 


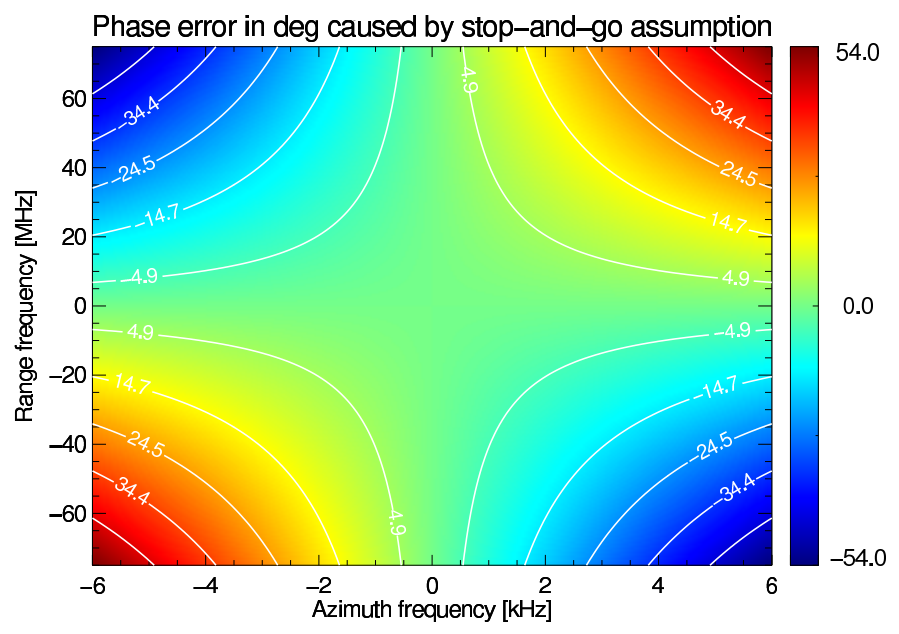

Fig. 16. Phase error due to the motion of the platform during the transmission of the chirp (instantaneous Doppler shift) for a range/azimuth frequency band of $150 \mathrm{MHz} / 12 \mathrm{kHz}$, corresponding to the typical spectral support of TerraSAR-X TOPS data. The duration of the transmitted pulse is $50 \mu$ s.

platform during the transmission of the pulse changes the instantaneous range frequency of the chirp signal, which is offset by an amount

$$
\delta f_{\mathrm{i}} \approx f_{\mathrm{D}}=-\frac{1}{\lambda} \cdot \frac{\partial r\left(t_{\mathrm{r}}\right)}{\partial t_{\mathrm{r}}}
$$

where the subscript $\mathrm{i}$ refers to instantaneous, D to Doppler, $t_{\mathrm{r}}$ is the fast-time, and $r\left(t_{\mathrm{r}}\right)$ is the two-way range history for a given target. One intuitive way to visualize this is by considering different azimuth-frequency phase ramps for different wavelengths. In the context of pulsed radars, the effect was already discussed in [25], where the analysis was done in terms of a range compression mismatch, and in the context of high-resolution SAR imaging in [20]. Eq. (12) can be approximated by a phase perturbation in the wavenumber domain of the form

$$
\Phi_{\mathrm{SG}}\left(f_{\mathrm{a}}, f_{\mathrm{r}}\right) \approx-2 \pi \cdot f_{\mathrm{a}} \cdot \frac{f_{\mathrm{r}}}{K_{\mathrm{r}}},
$$

where the subscript SG stands for stop-and-go, and $K_{\mathrm{r}}$ is the chirp rate of the transmitted signal.

B. SAR image formation mismatch due to the intra-pulse motion of the satellite and experimental results with TerraSAR$X$ data

For illustrative purposes, Fig. 16 shows the values of (13) over the support frequency band of a typical TerraSAR-X TOPS acquisition. Within the usually small Doppler band of TOPS images, the effect basically results in a shift in the range dimension caused by the range-frequency ramp. Neglecting this effect has no impact on the phase of the targets, but causes a shift in the range dimension approximately proportional to the Doppler centroid with which the target is observed, i.e.,

$$
\delta t_{\mathrm{r}} \approx \frac{f_{\mathrm{DC}}}{K_{\mathrm{r}}} .
$$

The dependence of this range shift on the Doppler centroid skews the focused bursts (cf. Fig. 18). In practice, the effect
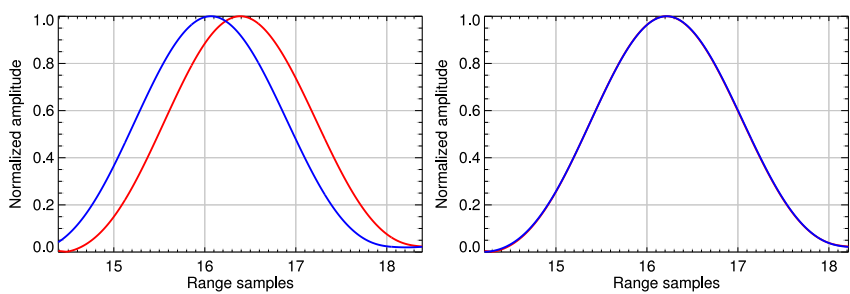

Fig. 17. Interpolated range response of a corner reflector located in the overlap region between two consecutive bursts. The lines correspond to the first burst (red) and the following burst (blue). Stop-and-go approximation uncorrected (left) and corrected (right).

of the motion of the platform is roughly space-invariant, which means it can be easily corrected in the wavenumber domain without additional burden (i.e., together with the bulk 2-D compression) using the complex conjugate of the phase function of (13) as suggested in [20].

Like in Section III, this effect can be observed using the overlap area between consecutive bursts. The corner reflectors placed in the overlap region show each an opposite displacement in range equal to (14). Therefore, the relative range shift between the responses of the two consecutive bursts takes the form

$$
\delta t_{\mathrm{r}, \mathrm{burst}} \approx \frac{\Delta f_{\mathrm{ovl}}}{K_{\mathrm{r}}}
$$

For the TerraSAR-X case, typical values for this differential range shift are of about 0.3 resolution cells. Fig. 17 shows this effect using the TerraSAR-X TOPS data over the DLR Oberpfaffenhofen test site, where the range response of one corner reflector located in the overlap area is shown for the two bursts involved, without (left) and with the proposed correction (right). Without the correction the shift is of about 0.3 resolution cells. Fig. 18 shows the deformation between two TOPS images of the same area, one processed with and the other without the proposed correction for the intrapulse motion of the platform. At the bottom of Fig. 18, the skew of the image lacking the correction as compared to the corrected one is evident and in agreement with (14). The range distortions caused by the intra-pulse motion of the platform mostly cancel out in interferometric pairs. ${ }^{8}$

\section{Corollary: The CaSe of Sentinel-1}

The Sentinel-1 mission is a constellation of two satellites (A and B units) each carrying an imaging C-band SAR instrument $(5.405 \mathrm{GHz})$ providing data continuity of ERS and ENVISAT SAR missions. Sentinel-1 is specifically designed to acquire systematically and provide routinely data and information products for the European Copernicus program for ocean, land and emergency services as well as national user services. In particular, the Sentinel-1 Interferometric Wide Swath mode (IW) and the Extra Wide Swath mode (EW) operate in TOPS to provide large swath widths of $250 \mathrm{~km}$ and 400

\footnotetext{
${ }^{8}$ Small residual radargrammetric shifts may still be present due to the lack of parallelism between the orbits of master and slave; these shifts, in the millimetric range for TanDEM-X, disappear once the correction of (13) is applied.
} 

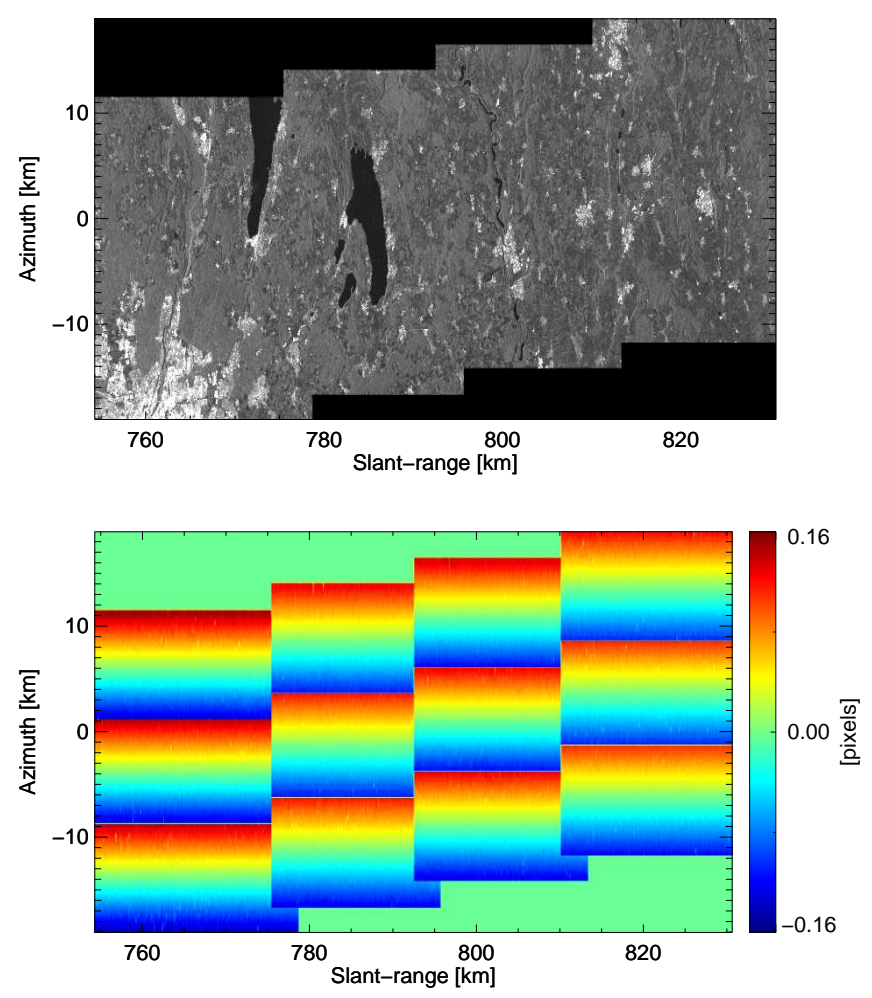

Fig. 18. Image deformation due to the stop-and-go approximation. TerraSAR$\mathrm{X}$ TOPS reflectivity image (top) over the test area in Oberpfaffenhofen, Munich. Estimated misregistration error in range (bottom) between the same images processed with and without the start-stop assumption, respectively. With the start-stop approximation, the image is clearly skewed as predicted by (14). Note that there is a large jump in the range coregistration error at the burst and the subswath edges.

TABLE II

KEPLERIAN ELEMENTS OF THE SENTINEL-1 ORBIT

\begin{tabular}{cc}
\hline Eccentricity & 0.001181 \\
Inclination & $98.12 \mathrm{deg}$ \\
Argument of perigee & $90.008 \mathrm{deg}$ \\
Ascending node & $250.409 \mathrm{deg}$ \\
Semimajor axis & $7070.978 \mathrm{~km}$ \\
\hline
\end{tabular}

$\mathrm{km},{ }^{9}$ respectively, with enhanced image performance (i.e., significantly reduced scalloping) as compared to traditional ScanSAR. The IW TOPS mode, the main mode of operations, enables a continuous data acquisition with global coverage.

In order to evaluate the impact of the potential distortions on Sentinel-1 TOPS images, analogous simulations as those described above for TerraSAR-X have been conducted. As in Section III, the results values have been computed using a numerical spaceborne SAR acquisition simulator; Table II lists the Keplerian parameters used for the simulation of the Sentinel-1 orbit. Table III provides the values of the key acquisition parameters used in the simulation for Sentinel-1, with the corresponding values of TerraSAR-X for comparison. The behavior of the effective velocity with respect to squint

${ }^{9}$ In IW mode, the size of the resolution cell is (range times azimuth) $5 \mathrm{~m}$ $\times 20 \mathrm{~m}$; in EW mode, $20 \mathrm{~m} \times 40 \mathrm{~m}$.
TABLE III

TOPS SIMULATION PARAMETERS FOR TERRASAR-X AND SENTINEL-1

\begin{tabular}{ccc}
\hline Radar satellite & TerraSAR-X & Sentinel-1 \\
Central frequency [GHz] & 9.65 & 5.405 \\
Wavelength [cm] & 3.1 & 5.55 \\
Transmitted chirp bandwidth [MHz] & 100 & 56.59 \\
Azimuth processed bandwidth [Hz] & 450 & 325 \\
Doppler shift overlap region [kHz] & 6 & 4.7 \\
Orbital tube diameter [m] & 250 & 100 \\
\hline
\end{tabular}

and topography is similar to the TerraSAR-X case, which yields in average smaller phase errors (about 80\%) in the SLCs due to the lower carrier frequency (combined with a higher orbit height) of Sentinel-1. The azimuth positioning errors are in average similar, whereas in range they are slightly smaller due to the smaller transmitted bandwidth and the similar duration of the radar pulses; this leads to range positioning errors at the edge of the bursts of about 0.13 resolution cells, and between consecutive bursts of about 0.25 resolution cells. Phase errors in interferometric TOPS pairs are expected to be, on average, 2.5 times smaller than those obtained with TerraSAR-X, due to both the smaller carrier frequency and the smaller orbital tube for Sentinel-1. As a consequence, the described effects have a negligible impact on the quality of the Sentinel-1 interferometric TOPS pairs acquired in both IW and EW modes.

\section{DISCUSSION}

The paper has presented the intrinsic distortions of TOPS SAR images due to the azimuth variation of the Doppler centroid within a burst. In particular, distortions in azimuth are caused by the local mismatch of the effective velocity of the radar due to squint and topography; distortions in range arise from the stop-and-go approximation. Compensation strategies for both effects have been discussed and validated using real TerraSAR-X TOPS data. The accommodation of squint and topography can be precisely achieved using the PASTA algorithm, which roughly reproduces the burden of an additional azimuth refocusing step. The correction of the stop-andgo approximation usually used in the SAR focusing kernel can be cheaply implemented after bulk 2-D compression. If not compensated, range and azimuth distortions reduce the achievable geolocation accuracy of TOPS SAR images. For interferometric applications, the analyzed effects might compromise the usability of TOPS SAR images for large baseline acquisitions or multi-mode coherent stack processing with SAR data acquired with very different observation geometries. In state-of-the-art missions like TerraSAR-X and Sentinel-1, these distortions have been found to have no significant impact on the TOPS interferometric image quality due to the usually small baselines.

\section{REFERENCES}

[1] F. De Zan and A. Monti Guarnieri, "TOPSAR: Terrain observation by progressive scans," IEEE Trans. Geosci. Remote Sens., vol. 44, no. 9, pp. 2352-2360, Sep. 2006.

[2] K. Tomiyasu, "Conceptual performance of a satellite borne, wide swath synthetic aperture radar," IEEE Trans. Geosci. Remote Sens., vol. 19, no. 2, pp. 108-116, Jan. 1981. 
[3] A. Meta, J. Mittermayer, P. Prats, R. Scheiber, and U. Steinbrecher, "TOPS imaging with TerraSAR-X: Mode design and performance analysis," IEEE Trans. Geosci. Remote Sens., vol. 48, no. 2, pp. 759769, Feb. 2010.

[4] R. Torres, P. Snoeij, D. Geudtner, D. Bibby, M. Davidson, E. Attema, P. Potin, B. Rommen, N. Floury, M. Brown, I. N. Traver, P. Deghaye, B. Duesmann, B. Rosich, N. Miranda, C. Bruno, M. L'Abbate, R. Croci, A. Pietropaolo, M. Huchler, and F. Rostan, "GMES Sentinel-1 mission," Remote Sensing of Environment, vol. 120, no. 0, pp. 9 - 24, 2012.

[5] G. Davidson, V. Mantle, B. Rabus, D. Williams, and D. Geudtner, "Implementation of TOPS mode on RADARSAT-2 in support of the Sentinel-1 mission," in Living Planet Symposium, Edinburgh, Scotland, 2013.

[6] A. Monti Guarnieri and C. Prati, "ScanSAR focusing and interferometry," IEEE Trans. Geosci. Remote Sens., vol. 34, no. 4, pp. 1029-1038, Jul. 1996.

[7] A. Moreira, J. Mittermayer, and R. Scheiber, "Extended chirp scaling algorithm for air- and spaceborne SAR data processing in stripmap and ScanSAR imaging modes," IEEE Trans. Geosci. Remote Sens., vol. 34, no. 5, pp. 1123-1136, Sep. 1996.

[8] R. Lanari, S. Hensley, and P. Rosen, "Chirp Z-transform based SPECAN approach for phase-preserving ScanSAR image generation," IEE Proc.Radar, sonar Navig., vol. 145, no. 5, pp. 254-261, Oct. 1998.

[9] P. Prats, R. Scheiber, A. Meta, and A. Moreira, "Processing of sliding spotlight and TOPS SAR data using baseband azimuth scaling," IEEE Trans. Geosci. Remote Sens., vol. 48, no. 2, pp. 770-780, Feb. 2010.

[10] G. Sun, M. Xing, Y. Wu, Y. Wu, and Z. Bao, "Sliding spotlight and TOPS SAR data processing without subaperture," IEEE Geoscience and Remote Sensing Letters, pp. 1 - 5, 2011.

[11] C. Y. Chang, M. Y. Jin, and J. C. Curlander, "Squint mode SAR processing algorithms," in Proc. IEEE International Geoscience and Remote Sensing Symposium (IGARSS'89), vol. 3, Vancouver, British Columbia, Canada, Jul. 10-14, 1989, pp. 1702-1706.

[12] A. Moreira and Y. Huang, "Airborne SAR processing of highly squinted data using a chirp scaling approach with integrated motion compensation," IEEE Trans. Geosci. Remote Sens., vol. 32, no. 5, pp. 1029-1040, Sep. 1994.

[13] G. W. Davidson, I. G. Cumming, and M. R. Ito, "A chirp scaling approach for processing squint mode SAR data," IEEE Trans. Aerosp. Electron. Syst., vol. 32, no. 1, pp. 121-133, Jan. 1996.

[14] G. Fornaro, E. Sansosti, R. Lanari, and M. Tesauro, "Role of processing geometry in SAR raw data focusing," IEEE Trans. Aerosp. Electron. Syst., vol. 38, no. 2, pp. 441-454, Apr. 2002.

[15] S. N. Madsen, "Motion compensation for ultra wide band SAR," in Proc. IEEE International Geoscience and Remote Sensing Symposium (IGARSS'01), vol. 3, Sidney, Australia, Jul. 9-13, 2001, pp. 1436-1438.

[16] K. Macedo and R. Scheiber, "Precise topography- and aperturedependent motion compensation for airborne SAR," IEEE Geosci. Remote Sens. Lett., vol. 2, no. 2, pp. 172-176, Apr. 2005.

[17] P. Prats, K. A. C. de Macedo, A. Reigber, R. Scheiber, and J. J. Mallorqui, "Comparison of topography- and aperture dependent motion compensation algorithms for airborne SAR," IEEE Geosci. Remote Sens. Lett., vol. 4, no. 3, pp. 349-353, Jul. 2007.

[18] H. Cantalloube and G. Krieger, "Elevation-dependent motion compensation for frequency-domain bistatic SAR image synthesis," in Proc. IGARSS, Barcelona, Spain, Jul. 23-27, 2007, pp. 2148-2151.

[19] M. Rodriguez-Cassola, P. Prats, G. Krieger, and A. Moreira, "Efficient time-domain image formation with precise topography accommodation for general bistatic SAR configurations," IEEE Trans. Aerosp. Electron. Syst., vol. 24, no. 3, pp. 218-223, Oct. 2011.

[20] P. Prats-Iraola, R. Scheiber, M. Rodriguez-Cassola, J. Mittermayer, S. Wollstadt, F. De Zan, B. Bräutigam, M. Schwerdt, A. Reigber, and A. Moreira, "On the processing of very high resolution spaceborne SAR data," IEEE Trans. Geosci. Remote Sens., accepted for publication.

[21] T. Kempff, H. Anglberger, and H. Suess, "Depth-of-focus issues on spaceborne very high resolution SAR," in Proc. IGARSS, Munich, Germany, Jul. 21-25, 2012.

[22] A. Meta, P. Hoogeboom, and L. P. Ligthart, "Signal processing in FMCW SAR,” IEEE Trans. Geosci. Remote Sens., vol. 45, no. 11, pp. 3519-3532, Nov. 2007.

[23] D. D'Aria and A. Monti Guarnieri, "High-resolution spaceborne SAR focusing by SVD-Stolt," IEEE Geosci. Remote Sens. Lett., vol. 4, no. 4, pp. 639-643, Oct. 2007.

[24] F. K. Li, D. N. Held, J. Curlander, and C. Wu, "Doppler parameter estimation for spaceborne synthetic aperture radars," IEEE Trans. Geosci. Remote Sens., vol. 23, no. 1, pp. 47-56, Jan. 1985.
[25] J. C. Curlander and R. N. McDonough, Synthetic Aperture Radar: Systems and Signal Processing. New York, USA: John Wiley \& Sons, 1991.

[26] I. G. Cumming and F. H. Wong, Digital Processing of Synthetic Aperture Radar Data. Algorithms and Implementation. Boston, London: Artech House, 2005.

[27] R. Stolt, "Migration by Fourier transform techniques," Geophysics, vol. 43, no. 1, pp. 49-76, 1978.

[28] P. Prats-Iraola, R. Scheiber, L. Marotti, S. Wollstadt, and A. Reigber, "TOPS interferometry with TerraSAR-X," IEEE Trans. Geosci. Remote Sens., vol. 50, no. 8, pp. 3179-3188, Aug. 2012.

[29] R. F. Hanssen, Radar Interferometry. Data Interpretation and Error Analysis. The Netherlands: Kluwer Academic Publishers, 2001.

[30] H. Breit, T. Fritz, U. Balss, M. Lachaise, A. Niedermeier, and M. Vonavka, "TerraSAR-X SAR processing and products," IEEE Trans. Geosci. Remote Sens., vol. 48, no. 2, pp. 727-740, Feb. 2010. 